\title{
Palliativmedizin und Interdisziplinarität Auftrag und Wirklichkeit
}

\author{
K.E. Clemens, Chr. Ostgathe, E. Klaschik
}

Abteilung für Anästhesiologie, Intensivmedizin, Schmerztherapie und Palliativmedizin, Malteser Krankenhaus Bonn, Universität Bonn

(Leitung: Prof. Dr. E. Klaschik)

klinikarzt 2005; $34(1+2): 11-14$

$\mathrm{P}$ alliativmedizin ist die Behandlung von Patienten, deren Erkrankung nicht heilbar, weit fortgeschritten, fortschreitend und lebensbegrenzend ist. Ihr Hauptziel ist die Verbesserung der Lebensqualität dieser Patienten. Um dies zu erreichen, gehört zur Palliativmedizin nicht nur die Berücksichtigung physischer Aspekte, wie zum Beispiel körperlich begründbarer Schmerz oder andere Symptome, sondern auch derjenigen aus dem psychischen, sozialen und spirituellen Bereich. Übergeordnetes Ziel einer derartigen ganzheitlichen Betreuung ist es, den Patienten bei weitestgehender Beschwerdefreiheit bis zu ihrem Tode ein Leben in Würde zu ermöglichen - mit Beistand von Angehörigen, Freunden, ehrenamtlichen und professionellen Begleitern.

Ein solch umfassendes Betreuungskonzept stellt vielfältige und weit reichende Anforderungen an das behandelnde Team, das multiprofessionell und zur interdisziplinären Zusammenarbeit fähig sein muss. Es reicht dabei nicht aus, wenn die verschiedenen Disziplinen eigenständig nebeneinander und unabhängig voneinander den Patienten betreuen. Vielmehr müssen die verschiedenen Berufsgruppen und Disziplinen ineinander greifen und sich gegenseitig einbeziehen.

Die Palliativmedizin hat ihren Ursprung in der modernen Hospizbewegung. Diese beruht auf einem ganzheitlichen Konzept für die Betreuung von schwer kranken und sterbenden Patienten. Im Vordergrund steht nicht die Lebensverlängerung, sondern das Erreichen der bestmöglichen Lebensqualität in der noch verbleibenden Zeit. Ein derartiges ganzheitliches Betreuungskonzept stellt vielfältige und weit reichende Anforderungen an das behandelnde Team, das zur interdisziplinären Zusammenarbeit fähig sein muss. Um dies sicherzustellen, müssen zunächst die Vertreter der involvierten Berufsgruppen über die entsprechende Haltung und Expertise verfügen. Dringend erforderlich sind daher die Verankerung der Palliativmedizin als Pflichtlehr- und -prüfungsfach in die Ausbildung von Medizinstudenten, eine intensivere Berücksichtigung der Palliativpflege in den Ausbildungsgängen pflegerischer Berufe sowie eine Vermittlung von palliativmedizinischen Basiskenntnissen inklusive Ethik und Kommunikation auch in allen anderen involvierten Berufsgruppen. Darüber hinaus darf die Forschung in der Palliativmedizin nicht weiterhin vernachlässigt werden, um eine Weiterentwicklung und Anerkennung dieser Fachdisziplin sicherzustellen. Vor dem Hintergrund, dass jeder Patient grundsätzlich ein Recht auf die bestmögliche medizinische Versorgung und Therapie hat, auch wenn eine kurative Behandlung nicht mehr möglich ist, sind sowohl die Ausweitung der klinischen Forschung als auch die deutliche Verbesserung des palliativmedizinischen Angebots ethische Verpflichtungen für unser Gesundheitssystem.

Voraussetzung dafür ist eine offene und zeitnahe Kommunikation innerhalb des Teams. Ist dieser Kommunikationsprozess im Team gestört, scheitert die Umsetzung der Palliativmedizin in einem wesentlichen Punkt.

\section{Was ist Palliativmedizin heute?}

Die 1994 gegründete Deutsche Gesellschaft für Palliativmedizin umschreibt in Anlehnung an die Definition der Weltgesundheitsorganisation (WHO) Palliativmedizin als Behandlung von Patienten mit einer nicht heilbaren, progredienten und weit fortgeschrittenen Erkrankung und begrenzter Lebenserwartung, für die das Hauptziel der Begleitung die Lebensqualität ist. Diese Definition beschränkt die palliativen Behandlungsmöglichkeiten nicht auf maligne Krankheiten, sondern schließt viele Patienten mit chronischen Leiden oder Behinderungen ein.

Obwohl die Linderung von Leiden die Aufgabe aller Ärzte und Fachgebiete war und ist - ganz gleich welche Ursache dazu geführt hat oder wie weit die Erkrankungen fortgeschritten sind - zielt die Palliativ- 


\section{Tab. 1 Das Gesamtkonzept der Palliativmedizin}

- exzellente Schmerz- und Symptomkontrolle

- Integration der psychischen, sozialen und seelsorgerischen Bedürfnisse der Patienten, der Angehörigen und des Behandlungsteams sowohl bei der Krankheit als auch beim Sterben und in der Zeit danach

- Akzeptanz des Todes als Teil des Lebens. Bei deutlicher Bejahung des Lebens soll der Tod weder bescheunigt noch hinausgezögert werden. Palliativmedizin ist eine eindeutige Absage an die aktive Sterbehilfe

- Kompetenz in den wichtigen Fragen der Kommunikation und Ethik

medizin hauptsächlich auf die Linderung von Leiden im Endstadium ab, wenn sich das Augenmerk der Behandlung ganz auf die Schaffung von Lebensqualität richtet und nicht mehr auf die Verlängerung des Lebens (4). Einem neueren Selbstverständnis der Palliativmedizin zufolge sollen die Möglichkeiten der Palliativmedizin Patienten und Angehörigen bei Bedarf aber auch schon ab dem Zeitpunkt der Diagnosestellung zur Verfügung gestellt werden können (3). Die palliativmedizinische Betreuung basiert auf der hohen Fachkompetenz, insbesondere in Schmerztherapie und Symptomkontrolle, sowie auf inter- und multidisziplinärer Zusammenarbeit unterschiedlicher Berufsgruppen wie Ärzten, Pflegenden, Seelsorgern, Psychologen, Trauerbegleitern, Physiotherapeuten, Sozialarbeitern und zusätzlich ehrenamtlichen Mitarbeitern.

Es ist die Einstellung gegenüber der Symptomkontrolle, welche die Palliativmedizin von der klassischen kurativen Medizin unterscheidet. Die Befreiung oder Linderung von Symptomen wird zum alles überragenden Mittelpunkt der Therapie (14). Als weiteres und nicht zu vernachlässigendes Element gilt die Kommunikation mit dem schwer kranken oder sterbenden Patienten und dessen Angehörigen. Aufrichtigkeit bei der Mitteilung „schlechter Nachrichten“ und Hilfestellung bei der Trauerverarbeitung sind hierfür Beispiele.

Die Rehabilitation des Kranken als dritter Baustein soll schließlich ein - an den verbliebenen Fähigkeiten gemessenes - „normales Leben“ ermöglichen, also zum Beispiel die Pflege sozialer Kontakte und die Ausübung lieb gewonnener $\mathrm{Ge}$ wohnheiten (14). Auch wenn die Palliativmedizin nach der oben dargestellten Definition nicht auf die Behandlung von Patienten mit unheilbaren Tumorerkrankungen beschränkt ist, sollte doch darauf hingewiesen werden, dass traditionsgemäß in den meisten Palliativeinrichtungen Tumorpatienten betreut werden.

Die Palliativmedizin schließt eine Chemotherapie, Strahlentherapie und/oder operative Therapie nicht aus. Voraussetzung ist aber, dass die Vorteile dieser Maßnahmen größer sind als deren potenzielle Nachteile $(7,12)$.

Aufgabe und Ziel der Palliativmedizin ist es, Unterstützung anzubieten, damit der Patient die bestmögliche Lebensqualität in der ihm verbleibenden Zeit erreichen kann. Dies wird durch die Kooperation von kompetenten palliativen Einrichtungen mit Hausärzten, Sozialstationen und Krankenhausstationen angestrebt, indem eine optimale Behandlung rund um die Uhr überall dort gesichert werden soll, wo diese Patienten betreut werden. Dabei ist vorrangig, dem Patienten die Möglichkeit zu geben, selbst zu entscheiden, ob er zu Hause oder in stationären Einrichtungen versorgt werden will.

Die Integration dieser Themen in die medizinische Lehre und Forschung ist eine Hauptaufgabe für die kommenden Jahre (2).

\section{Gegenwärtiger Stand in Deutschland}

Krebs ist ein weltweites Problem. Pro Jahr sterben vier bis fünf Millionen Menschen an den Folgen ihrer Tumorerkrankung. In Deutschland sind dies jährlich etwa 220000 Menschen, damit gehen 25\% aller Todesfälle zu Lasten eines Karzinoms (11, 13). Von den jährlich 300000 Neuerkrankungen an Krebs in Deutschland können bislang nur 45\% durch primäre chirurgische, strahlentherapeutische oder chemotherapeutische Behandlungsstrategien geheilt werden (5). Weitere 15\% der primär behandelten Patienten erleiden ein inkurables Erkrankungsrezidiv (5). In den letzten 20-30 Jahren haben neue Behandlungsmöglichkeiten die Aussicht auf Heilung für viele Krebskrankheiten nicht bessern können (1).

Diese Gesamtentwicklung hat uns zur Entwicklung neuer Konzepte in der Behandlung von schwer kranken Tumorpatienten verpflichtet, die keine Aussicht auf Heilung haben und bei welchen erfahrungsgemäß stärkste Schmerzen und andere körperliche Symptome, Lebenskrisen, Angst und Leiden im Mittelpunkt ihrer letzten Monate und Tage stehen können. Die Weltgesundheitsorganisation (WHO) räumt deswegen der Palliativmedizin höchste Priorität ein (12).

Die Palliativmedizin, als lindernder Ansatz wahrscheinlich die älteste medizinische Disziplin überhaupt, setzt heute auf medizinische und wissenschaftliche Fortschritte der letzten Jahrzehnte in der Schmerztherapie und Symptomkontrolle sowie auf neue Erkenntnisse über die elementaren Bedürfnisse Schwerstkranker und Sterbender. Wiederentdeckt und mit hoher Priorität werden Kommunikation, Ethik, Mitmenschlichkeit, Teamarbeit und der Mensch in seiner ganzheitlichen Dimension in die moderne Schmerztherapie und Symptomkontrolle im Rahmen der interdisziplinären palliativen Therapie eingebunden und berücksichtigt.

Obwohl die Palliativmedizin in Deutschland seit Anfang der 1980er Jahre praktiziert wird und seit Anfang der 1990er Jahre erhebliche Fortschritte gemacht hat, bestand im Jahr 2003 mit einem Angebot von nur 9,1 Palliativbetten pro einer Million Einwohner eine deutliche Unterversorgung. Zusätzlich gab es 12,4 Hospizbetten pro Million Einwohner - insgesamt also 21,5 Betten pro eine Million Einwohner (10). Wünschenswert wäre aber gut das Doppelte (6). Nur 3-4\% der Patienten, die an Krebserkrankungen sterben, können damit auf Palliativstationen betreut werden. Hochrechnungen haben ergeben, dass zirka 17-18\% nicht heilbarer Tumorpatienten eine vorübergehende sta- 
tionäre Behandlung auf solchen Stationen benötigen.

Die stationäre Versorgung dieser Patientengruppe wird im Besonderen an den deutschen Universitäten vernachlässigt. Von den 93 Palliativstationen, die wir derzeit in Deutschland haben, sind nur acht an Universitäten angegliedert. $\mathrm{Zu}$ der derzeit absolut zu niedrigen Bettenzahl im Palliativbereich kommt eine inhomogene Verteilung dieser Betten hinzu.

Beispielhaft sei das Land Nordrhein-Westfalen genannt, wo die Bettenverteilung in den Regierungsbezirken zwischen einem und elf Betten pro einer Million Einwohner variiert. Wenn Leiden in $\mathrm{Zu}-$ kunft umfassend gelindert und Palliativmedizin als Lebensperspektive und als Alternative zur aktiven Sterbehilfe betrachtet werden soll, dann muss ein flächen- und bedarfsdeckendes palliativmedizinisches Netzwerk aufgebaut werden (9).

\section{Ausbildung und Standard}

In Deutschland gibt es inzwischen von der Deutschen Gesellschaft für Palliativmedizin (DGP) empfohlene Curricula für Medizinstudenten, Ärzte, Sozialarbeiter, Seelsorger und Krankenpflegekräfte. Inhalte der Weiterbildung sind: Schmerztherapie, Symptomkontrolle, konservative, interventionelle und operative Onkologie, Strahlentherapie, Ethik, Kommunikation, Sterben, Tod und Trauer, Seelsorge, Psychologie und Betreuung des therapeutischen Teams.

Bisherige Defizite in der Ausund Fortbildung sind unverkennbar. Unbestritten ist auch der aufgrund der demografischen Entwicklung und sich damit erhöhenden Inzidenz von Tumorerkrankungen zu erwartende weiter steigende Bedarf an Palliativmedizin. Daher müssen die gesundheits- und bildungspolitisch Verantwortlichen sowie die Lehrenden der einzelnen Fachdisziplinen erkennen, dass Palliativmedizin in die ärztliche Approbationsordnung und somit entsprechend in die Studienordnungen und Lehrpläne der medizinischen Fakultäten integriert werden muss (10).
Die im Jahr 2002 im Bundestag verabschiedete neue Approbationsordnung für Ärzte enthält zwar erstmalig den Begriff Palliativmedizin. Sie wurde aber leider nicht als Pflichtlehr- und -prüfungsfach berücksichtigt, sondern nur als optionales Fach. Damit wurde eine große Chance vertan, die zukünftige Arztgeneration in der Universitätsausbildung mit den Grundprinzipien der Palliativmedizin vertraut zu machen. Nur an den Universitäten Bonn und München gehört das Fach seit dem Jahr 2004 zu den Pflichtveranstaltungen.

Nicht zuletzt wegen der immer wieder aufflackernden Diskussion um die Legalisierung der aktiven Sterbehilfe muss mit der Förderung der Palliativmedizin nachhaltig eine Alternative in unserem Gesundheitssystem verankert werden, die für unsere Patienten eine Lebensperspektive aufzeigt.

\section{Palliativmedizin - praktische Umsetzung der Interdisziplinarität} im ambulanten Bereich anhand eines Patientenbeispiels

E in etwa 65-jähriger Patient erkrankt einige Monate nach seiner Pensionierung an einem - Tumor. Er ist verheiratet, die Ehe ist kinderlos geblieben. Trotz intensiver tumorhemmender Therapie schreitet die Erkrankung in den Monaten nach der Diagnosestellung rasch voran. Der Patient - enttäuscht von der medizinischen Betreuung - beschließt, alle auf Heilung ausgerichteten Maßnahmen abzubrechen. Er möchte unter gar keinen Umständen mehr in ein Krankenhaus.

In den folgenden Wochen wird der Patient zunehmend bettlägerig, seine Ehefrau pflegt ihn liebevoll zu Hause. Wegen der sehr schmerzhaften Hautmetastasen, die mittlerweile am gesamten Körper aufgetreten sind, wird die Pflege - insbesondere die Körperpflege (Betten, Waschen) - für den Patienten, aber auch für seine Frau zur Qual. Der ambulante Palliativdienst wird zur Unterstützung sowie Optimierung der Schmerztherapie mit einbezogen. Hierdurch ist der Patient in Ruhe fast schmerzfrei. Doch trotz aller zur Verfügung stehender Maßnahmen (bis hin zur Körperpflege mit Betten und Waschen in Kurznarkose) bleiben die Bewegungen und Lagerungsmaßnahmen bei der Körperpflege für den Patienten sehr schmerzhaft.

Der Schwerpunkt der Betreuung verschiebt sich zunehmend von der Schmerztherapie und Symptomkontrolle zu einer intensiven Begleitung von Patient und Ehefrau. Viele Gespräche unterstützen die Auseinandersetzung mit der Dynamik der Erkrankung und den sich daraus ergebenden Folgen. Für beide ist die Zusicherung beruhigend, zu jedem Zeitpunkt Hilfe bekommen zu können. Es werden Möglichkeiten besprochen, wie dem Leiden medizinisch zu begegnen sei, falls sich die Situation weiter zuspitzen sollte. Für diesen Fall wird mit dem Patienten und seiner Ehefrau eine Sedierung besprochen.

Mehrfach im Verlauf der letzten Lebenstage wird bei krisenhaften Veränderungen erwogen, ob nicht eine weitere Betreuung auf der Palliativstation sinnvoll sei. Aber der Patient besteht darauf, zu Hause zu bleiben. In einem Gespräch äußert der Hausarzt gegenüber dem ambulanten Palliativdienst, ob nicht eine aktive Sterbehilfe sinnvoll wäre. Er fühle sich mit der Behandlung überfordert, sei aber froh über die Einbindung des Palliativdienstes. Im weiteren Verlauf der Betreuung wird der Patient zunehmend schwächer und schläfriger. Kurze Phasen der Unruhe können durch die Gabe von Beruhigungsmitteln gelindert werden. Durch den Verzicht auf weitere Lagerungsmaßnahmen und intensive Körperpflege bleibt der Patient weitestgehend schmerzfrei. Etwa zehn Tage nach Betreuungsbeginn verstirbt der Patient zu Hause im Beisein seiner Ehefrau - ruhig und gut symptomkontrolliert. 
Ins Netz gegangen

Ins Netz gegangen

www.hospiz.net

Die Bundesgemeinschaft Hospiz e.V. zur Förderung von ambulanten, teilstationären und stationären Hospizen und Palliativmedizin (BAG), die Landesgemeinschaften und Links zu einigen anderen auch internationalen - Organisationen (z.B. Internationale Gesellschaft für Sterbebeistand und Lebensbegleitung e.V. - IGSL) oder Initiativen finden Sie unter dieser Internetadresse. Über ein eMailAdressbuch können Sie direkt Kontakt zu diversen Hospizdiensten aufnehmen. Integriert sind zudem unter anderem ein Stellenmarkt, eine Linksammlung bzw. eine Sammlung von Publikationen (zu finden unter dem Link "Forum Hospiz“) oder gesetzlicher Regelungen zum Hospizbereich, die stets auf dem Laufenden gehalten werden.

\section{www.hospize.de}

Wissenswertes über die Hospizarbeit in Deutschland finden sich auf der Homepage der Deutschen Hospiz Stiftung, die in Deutschland als Patientenschutzorganisation für die Interessen der Schwerstkranken und Sterbenden kämpft. Über das Schmerz- und Hospiztelefon beispielsweise steht ein multiprofessionelles Team für Fragen oder eine Beratung zur Verfügung. Außerdem finden Sie hier - nach Postleitzahlen geordnet - eine aktuelle Liste (Stand Januar 2005) der Hospizdienste in Deutschland.

\section{www.deutscher-kinderhospizverein.de}

Die Begleitung der betroffenen Familien mit Anlaufstellen an verschiedenen Orten in Deutschland ist das Hauptanliegen des Deutschen Kinderhospizvereins e.V. Hier finden betroffene Eltern zum Beispiel die Geschichten von drei Familien, eine Bücherliste oder eine Aufstellung von Fachartikeln, aber auch Links zu Selbsthilfegruppen unterschiedlicher Krankheiten oder zum Umgang mit Tod und Trauer.

\section{www.patientenverfuegung.de}

Der Humanistische Verband Deutschlands bietet umfassende Informationen rund um das schwierige Thema Patientenverfügungen. Auf seiner Internetseite können sich Interessierte informieren, worauf beim Verfassen einer Patientenverfügung zu achten ist, welches Modell am besten „passt“ bzw. wie verbindlich solche Verfügungen tatsächlich sind. Zudem stehen Musterformulare für Vollmachten für finanzielle und rechtsgeschäftliche bzw. für medizinische und gesundheitliche Angelegenheiten zum Download zur Verfügung.

\section{In diesem Monat}

und Feingefühl die Konsequenzen ihrer Erkrankung deutlich zu machen. Es ist nicht mit der ärztlichen Ethik zu vereinbaren, einem schwer kranken Patienten die Möglichkeit zu nehmen, sich mit seinem bevorstehenden Lebensende auseinanderzusetzen, oder ihn „um jeden Preis" weiter zu therapieren. Der Versuch einer Entscheidung auszuweichen, der in der medizinischen Praxis nicht selten anzutreffen ist, kann für den Patienten und seine Angehörigen folgenschwer sein. Die Wahrheit am Krankenbett bedeutet keineswegs eine Ankündigung des Sterbens, sondern den Beginn eines Prozesses, der die Sinnerschließung des Sterbens als Teil des Lebens zum Inhalt hat.

Eine ehrliche Auseinandersetzung mit Tod und Sterben ist in unserem Kulturkreis immer noch ungewöhnlich. Das Sterben vollzieht sich nicht mehr zu Hause im Kreise der Familie. Noch hat das öffentliche Bewusstsein keine Trendwende im Umgang mit Sterben und Tod erreicht. Das zeigt sich besonders daran, dass es auch in Deutschland, ähnlich wie in den Niederlanden oder Belgien, zu einer breiten $\mathrm{Zu}$ stimmung zur aktiven Sterbehilfe gekommen ist. Die Entwicklung der Diskussion über eine eventuelle Legalisierung der aktiven Sterbehilfe fordert dazu heraus, eine andere Antwort auf das Leid Schwerstkranker und Sterbender zu geben. Diese Antwort heißt Palliativmedizin. Dem unerträglichen Leiden kann mit Zuwendung zum Menschen begegnet werden: Palliativmedizin ist aktive Lebenshilfe.

\section{Palliative Medicine and Interdisci- plinary Considerations -Theory and Practice}

Palliative medicine arose out of the modern hospice movement. This latter is based on a holistic concept for the care of seriously ill and dying patients. The overriding aim is not the prolongation of life, but ensuring the best possible quality of life in the remaining life time of the patient. Such a holistic concept of care makes manifold and extensive demands on the care-providing team, which must be capable of interdisciplinary coopera- tion. To ensure this, the representatives of the professional groups involved must have the appropriate expertise. To ensure this, there is an urgent need to incorporate palliative medicine in the teaching curriculum for medical students, greater emphasis on palliative care in the training of nurses, and the teaching of palliative medical basic knowledge, including ethical aspects and communication with all other relevant occupational groups. Furthermore, research into palliative medicine must no longer be assigned to the back burner, so as to ensure the further progress and recognition of this specialty. In view of the fact that every patient has a fundamental right to the best medical care and treatment, even if a cure is no longer possible, both the expansion of clinical research and an appreciable improvement in the palliative-medical resources are obligations that have to be met by our health care system.

\section{Key Words}

palliative medicine - quality of life holistic concept for care - interdisciplinary cooperation

\section{Literatur bei der Redaktion / im Internet unter www.klinikarzt.info}

\author{
Anschrift für die Verfasser \\ Dr. Katri Elina Clemens \\ Abteilung für Anästhesiologie, \\ Intensivmedizin, Schmerztherapie \\ und Palliativmedizin \\ Malteser Krankenhaus Bonn \\ Von-Hompesch-Str. 11 \\ 53123 Bonn
}

